\title{
Quadriceps tendon rupture. Anchor or transosseous suture? A systematic review
}

\author{
M. Corÿdon Hochheim¹, E. M. Bartels ${ }^{2}$, J. Vestergård Iversen ${ }^{3}$ \\ 1 University of Copenhagen, Denmark \\ 2 The Parker Institute and Department of Neurology, Copenhagen University Hospital, Bispebjerg and \\ Frederiksberg, Copenhagen N, Denmark \\ ${ }^{3}$ Department of Orthopaedic Surgery, Zealand University Hospital, Køge, Denmark
}

\author{
CORRESPONDING AUTHOR: \\ Martin Corÿdon Hochheim, \\ University of Copenhagen, \\ 2650 Hvidovre, Denmark \\ Phone: +4527218682 \\ E-mail: hochheim@live.dk \\ DOI: \\ 10.32098/mltj.03.2019.09
}

LEVEL OF EVIDENCE: $2 A$

\begin{abstract}
SUMMARY
Introduction. When dealing with ruptures at the tendon-bone junction either transosseous sutures(TS) or anchor fixation(AF) are the most well described methods of repair. The aim of this study was to compare these two surgical methods by carrying out a systematic review of the existing literature.

Methods. A systematic search of bibliographic databases to identify studies using either TS or AF for patients with QTR, with outcome measures being either range of motion(ROM), extension lag(EL), re-rupture(RR) and/or Lysholm score(LS).

Results. The AF group included 3 studies containing 45 patients. The postoperative outcomes reported were a ROM average of $128,5^{\circ}$, and 3 EL $(6,7 \%)$ and 2 RR were reported( $4,4 \%$ ). 2 postoperative LS showed an average of 88,9 .

The TS group included 2 studies containing 32 patients. One study reported an average ROM postoperatively of $138^{\circ}$, one a ROM under $120^{\circ}$ postoperatively in 2 out of 14 QTRs, 3 EL (13\%), no RR and one postoperative LS of 94 in 8 patients.

Conclusion. This study implies a slight postoperative advantage when using transosseous sutures compared to anchor fixation. However, given a low patient count and a lack of statistical power, no conclusion on the optimal surgical method for QTR can be made.
\end{abstract}

\section{KEY WORDS}

Knee; Quadriceps tendon rupture; Suture anchor; Transosseus suture

\section{INTRODUCTION}

Quadriceps tendon rupture(QTR) at the bone-tendon junction is an invalidating injury often seen in middle-aged men over 40 years of age (1). However, it also occurs in younger athletes $(2,3)$. The risk of QTR is increased by certain risk factors (4). Despite of these risk factors, the incidence remains low. According to Clayton et. al. the incidence of QTR in and around Edinburgh in Great Britain is $1,37 / 100,000(5)$.

The injury mechanism is typically described as an eccentric contraction of the knee when trying to prevent a fall $(4,6)$, although spontaneous ruptures do occur (4). Typical findings are pain above the knee cap, a palpable supra-patellar gap and inability to actively extend the knee $(7,8)$

Several surgical techniques have been suggested in the literature (7,9-11). However transosseous sutures and anchor fixation are the most well described when dealing with ruptures at the tendon-bone junction. Since being reported in the mid 1950's (12), using transosseous sutures to reinsert the tendon through patella drill holes has remained the primary option when dealing with QTR $(1,13)$.

The use of suture anchors drilled into the patella when reinserting the quadriceps tendon(QT) was first described in year 2000 by Maniscalco et al. (14). This has lately been proposed as a good alternative to transosseous sutures given strong bio mechanical properties and furthermore an easier approach $(15,16)$. Advantages such as reduced operation time, the use of a smaller skin incision, as well as earlier rehabilitation have also been mentioned (17). However disadvantages regarding price and risk of infection due to the use of a foreign body must be taken into consideration. A need for further studies comparing the two interventions 
has been pointed out $(17,18)$, given that re-fixation of the QT using transosseous sutures is thoroughly tested and possible long-term complications are known (18).

To our knowledge no systematic review has been published comparing the outcome of transosseous sutures to the outcome of anchor fixation when reinserting the QT. The aim of this study was to review the current literature on the two methods, and if possible carry out a meta-analysis focusing on postoperative outcome, with our hypothesis being a postoperative advantage when using suture anchors.

Hopefully this review will be able to shed some light on the surgical dilemma when choosing treatment for this injury.

\section{MATERIALS AND METHODS}

A systematic literature search in bibliographic databases was carried out with a pre-defined selection strategy according to pre-specified eligibility criteria. The procedure was defined by a protocol following the standards of the Cochrane Collaboration (http://www. cochrane-handbook.org/) which was made publicly available via PROSPERO (www.crd.york.ac.uk/PROSPERO/) (CRD42018088738) ) prior to carrying out the search and data handling.

\section{Literature search}

The bibliographic databases: MEDLINE via PubMed from 1966, EMBASE via Ovid from 1974, Web of Science from 1900 and The Cochrane Central Register of Controlled Trials, all until 16th January 2019 were searched.

The search strategy was ((quadriceps tendon OR knee) AND (suture anchor* OR transosseous suture* OR trans-osseous suture* OR patella drill bole*))

In Medline, EMBASE and Cochrane this was searched as $\mathrm{TI} / \mathrm{AB}$, and in Web of Science as free text. References from selected studies and relevant reviews were also scrutinized for further eligible studies.

\section{Inclusion criteria}

Studies were included if they met the following criteria:

1. The study population consisted of a minimum of 8 men and/or women aged $\geq 18$ years, and diagnosed with unilateral or bilateral QTR.

2. The diagnosis resulted in a surgical intervention with either the use of anchor fixation or transosseous sutures through patella drill holes.

3. The outcome measure was either extension lag and/ or range of motion and/or re-rupture and/or Lysholm score(19). For the Lysholm Score (scale 0-100), a higher score indicates healthier condition.

4. The outcome was well described and there was a minimum follow-up period of 12 weeks.

\section{Exclusion criteria}

1. QTR as a complication to total knee arthroplasty.

2. A diagnosis of mid tendon rupture resulting in end-toend tendon sutures.

3. A diagnosis of QTR in combination with a fracture/avulsion in relation to the knee joint.

4. Surgical intervention of each patient not clear or differing from the two interventions examined in this study.

\section{Selection of studies}

Two reviewers (MCH supported by JVI) assessed all retrieved studies, first by title and abstract, and further in full-text if positive evaluation at abstract level, to identify those that fulfilled the inclusion criteria. Disagreement concerning study inclusion or exclusion was resolved by consensus with a third reviewer (EMB).

\section{DATA ANALYSIS}

Two reviewers (MCH supported by JVI) extracted the following parameters from the included studies: study design; population size; follow-up period; characteristics of the study population (gender, age); surgical intervention; and postoperative outcome measures.

\section{STATISTICS}

Due to insufficient data, especially too few studies, the planned statistics and meta-analysis could not be carried out. However a Coleman Methodology Score(CMS)(20) was calculated for each study to assess the methodology of each of the included studies. The CMS is composed of 10 categories describing characteristics of the study in question and reported surgical outcomes with a maximum of 100 points.

\section{REGISTRATION AND FUNDING}

The protocol was uploaded to PROSPERO, the international prospective register of systematic reviews, and approved on the 14.02.2018. Registration number: CRD42018088738.

EMB was supported by a grant to the Parker Institute from the OAK foundation (OCAY-13-309). 


\section{RESULTS}

\section{Selection}

The literature search identified 137 articles when duplicates were removed (figure 1).

Out of these, 86 were excluded based on title and abstract. This was mainly due to lack of a QTR diagnosis or absence of a surgical intervention. The remaining studies were further scrutinized in full-text and an additional 39 studies were excluded. The main reasons for exclusion were either an unclear description of patients or surgical procedures, or inclusion of less than 8 patients, thereby being a multi-case study according our set definition. During data extraction a further 7 studies were excluded due to either an insufficient description of their method and/or their results.

Finally, 5 studies were divided into two groups according the intervention. One study included both interventions. The outcomes of the different studies using anchor fixation or transosseous sutures, respectively, are listed in Table II and III.

Due to the lack of randomized controlled studies the Cochrane Collaboration's tool for assessing risk of bias could not be applied.

Studies by Konrath et al.(21), Popov et al.(22), Vainionpää et al.(23), O'Shea et al.(24), Ramseier et al.(25), Boudissa et al.(26), Rasul et al.(27) were all excluded due to lack of sufficient description of method and/or outcome.

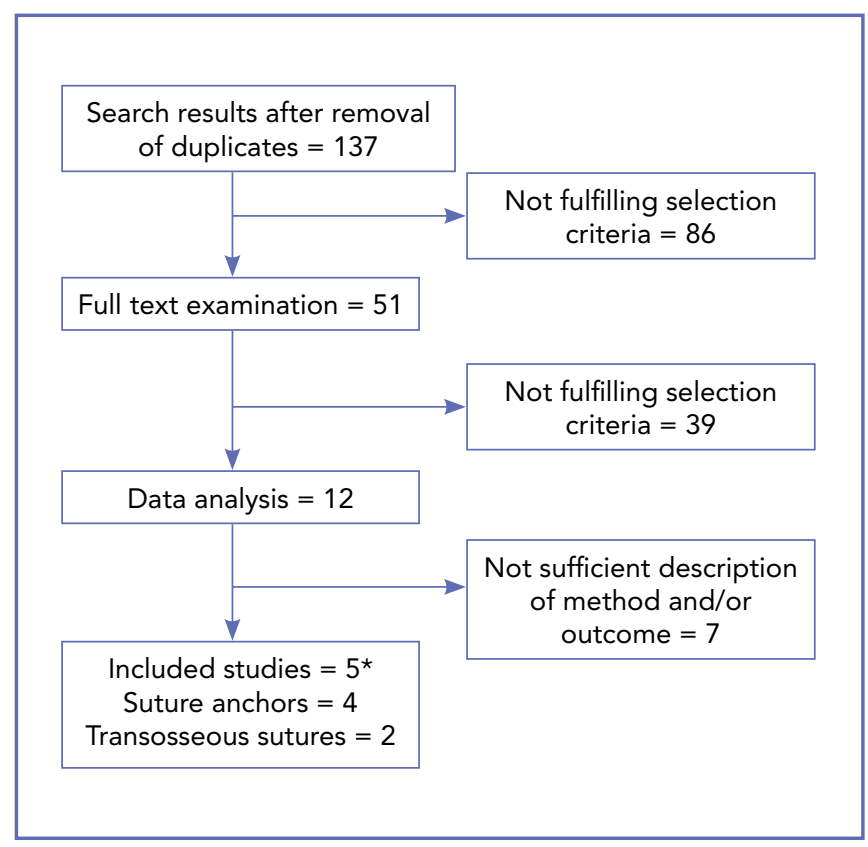

Figure 1. Selection process of included studies. *1 study included both interventions

\section{STUDY CHARACTERISTICS}

The anchor fixation group (table II) consisted of 81 patients from 4 studies $(13,28-30)$. A total of 81 QTRs were reported with 78 patients available for follow-up. The group consisted of 68 men and 10 women, with a mean age of 60,1 years (table I).

The transosseous suture group (table III) consisted of 32 patients from 2 studies $(29,31)$. A total of 23 QTRs were reported with 23 patients available for follow-up. The group consisted of 29 men and 3 women with a mean age of 58 years (table I).

\section{ANCHOR FIXATION}

Table II shows the results from studies using anchor fixation as a surgical approach for QTR.

All 4 studies reported ROM and re-rupture, and 3 out of 4 reported extension lag as an outcome measure. 2 out of 4 reported a postoperative Lysholm score. All 4 studies reported a postoperative ROM. In average, this was $123^{\circ}$ in 81 ruptures. 3 extension lags $(6,7 \%), 1$ in 3 of the studies, and 2 re-ruptures were reported (2,5\%). 2 postoperative Lysholm scores were reported with a weighted average of 88,9 out of 100 .

The surgical procedures are described in table III. For the postoperative regime Plesser et al.(29) allowed restricted active motion of the injured leg after 2 weeks, $40^{\circ}$ after 2 weeks and $60^{\circ}$ after 4 weeks, respectively. In opposition to this, Huleatt et al.(30), Brossard et al.(13) and Mille et al.(28) immobilized their patients in full extension for 6 weeks, with only passive motion and protected weight bearing. CMS is given for all studies in Table II.

\section{TRANSOSSEOUS SUTURES}

Table II shows the included studies using transosseous sutures as a surgical approach for QTR.

Both studies reported ROM, extension lag and re-ruptures as an outcome measure. Only Plesser et al.(29) reported a Lysholm score. De Baere et al.(31) reported the outcomes as ROM above or below $120^{\circ}$.

Table I. Patient characteristics

\begin{tabular}{llll}
\hline Intervention & M:F & $\begin{array}{l}\text { Mean age } \\
\text { (years) }\end{array}$ & $\begin{array}{l}\text { Mean follow-up } \\
\text { (months) }\end{array}$ \\
\hline Anchor fixation & $68: 10$ & 60,1 & 33.9 \\
\hline $\begin{array}{l}\text { Transosseous } \\
\text { sutures }\end{array}$ & $29: 3$ & 57,7 & 64,8 \\
\hline
\end{tabular}

M: Male; F: Female 
Table II. Characteristics and outcomes of included studies.

\begin{tabular}{|c|c|c|c|c|c|c|c|c|c|}
\hline Study & Surgical Method & Year & Type & QTRs & EL & $\begin{array}{l}\text { Av. } \\
\text { ROM }^{\circ}\end{array}$ & $\mathbf{R R}$ & Lysholm & CMS \\
\hline Huleatt et al. (30) & Anchor fixation & 2018 & Retrospective study & 36 & - & $116^{\circ}$ & 0 & - & 42 \\
\hline Brossard et al. (13) & Anchor fixation & 2017 & Retrospective study & $25(22)^{* *}$ & 1 & $128,2^{\circ}$ & 0 & 92 & 54 \\
\hline Plesser et al. (28) & $\begin{array}{l}\text { Transosseous } \\
\text { sutures }\end{array}$ & 2018 & Retrospective study & 8 & 0 & $138^{\circ}$ & 0 & 94 & 52 \\
\hline De Baere et al. (29) & $\begin{array}{l}\text { Transosseous } \\
\text { sutures }\end{array}$ & 2002 & Retrospective study & 15 & 3 & $(2) * *$ & 0 & - & 35 \\
\hline
\end{tabular}

QTRs: Quadriceps tenson ruptures. EL: Extension lag. ROM: Range of motion. RR: Re-rupture. Lysholm: Lysholm score(0-100). CMS: Coleman Methodology Score. *22 patients were included with 3 suffering from bilateral ruptures. **De Baere et al. only reported ROM as above or below $120^{\circ}$, with the reported result being the number of postoperative ROM under $120^{\circ}$.

Table III. Overview of the surgical procedures in the included studies

\begin{tabular}{|c|c|c|c|}
\hline Study & Year & Type & Description of surgical procedure \\
\hline Huleatt et al.(30) & 2018 & Anchor fixation & $\begin{array}{l}\text { 2-3 anchors with modified Mason-Allen sutures are placed using } 1 \text { FiberTape } \\
\text { and } 1 \text { FiberWire per anchor. } 4.75 \text { SwiveLock anchors are used. Either a } \\
\text { knotless technique with } 1 \text { FiberTape and } 1 \text { FiberWire suture through the tip } \\
\text { of each anchor or a knot-tied technique with sutures placed and a similar } \\
\text { modified Mason-Allen manner stitch pattern applied. }\end{array}$ \\
\hline Plesser et al.(28) & 2018 & Anchor fixation & $\begin{array}{l}3 \text { pilot holes in the proximal patella. } 35,5 \mathrm{~mm} \text { titanium corkscrew armed with } \\
2 \text { strands of FiberWire og two of TigerWire. A Mason-Allen stitch pattern was } \\
\text { used. }\end{array}$ \\
\hline Brossard et al.(13) & 2017 & Anchor fixation & $\begin{array}{l}\text { Modified Bunnell sutures using 2-3 suture anchors. } 2 \text { types of anchors: } 5.0 \\
\text { titanium corkscrew or } 5.5 \mathrm{~mm} \text { Healix Ti. }\end{array}$ \\
\hline Mille et al.(27) & 2015 & Anchor fixation & $\begin{array}{l}3 \text { barbed or threaded anchors made of titanium or resorbable material, } 5 \text { to } \\
6.5 \mathrm{~mm} \text { in diameter loaded with double needles. }\end{array}$ \\
\hline Plesser et al.(28) & 2018 & Transosseous sutures & $\begin{array}{l}\text { Drilling 3-4 longitudinal tunnels through the patella and suturing the tendon } \\
\text { using either 3\% Ethibond, polydioxane or FiberWire. } 3 \text { suture pattern } \\
\text { techniques were used: Mason-Allen, Krackow and Kessler-Kirchmayr. }\end{array}$ \\
\hline
\end{tabular}

Plesser et al.(29) reported an average ROM postoperatively, being $138^{\circ}$ averaged from 8 QTRs. De Baere et al.(31) reported a $\mathrm{ROM}$ under $120^{\circ}$ postoperatively in 2 out of 14 QTRs. 3 extension lags were reported out of a total of 23 QTRs (13\%). No re-ruptures were reported. Plesser et al.(29) reported a Lysholm score of 94 in 8 patients.

The surgical procedures are described in table III. The postoperative regime of the 2 studies did not differ substantially. Both studies immobilized their patients postoperatively for a period of 4,5-8 weeks. CMS is given for all studies in Table III.

\section{DISCUSSION}

Previously, when reinserting the QT at the tendon-bone junction transosseous sutures have been the golden standard(32) for treatment. Our findings showed a slight postoperative advantage when dealing with ruptures at the tendonbone junction and using transosseous sutures compared to anchor fixation. This does not support our hypothesis of suture anchors being superior, which was based on the use of anchor sutures becoming more frequent (33) and being recommended as a good alternative to transosseous sutures (14), especially in terms of better biomechanical results (16). 
When comparing refixation of the QT with suture anchors to transosseous sutures, suture anchors may well have a biomechanical advantage $(15,16)$. Furthermore there may be operative advantages such as reduced operation time, the use of a smaller skin incision and earlier rehabilitation $(14,17)$. However, with unknown long-term complications and a higher price compared to the transosseous sutures (18) anchor fixation is probably not cost beneficial.

The inclusion and exclusion criteria in the included studies were based on the current knowledge on QTR. Only ruptures at the tendon-bone junction were included, as bony avulsions or mid-tendon ruptures were not indications of any of the repair methods examined in this study (6). To narrow down the etiology, QTR as a complication to total knee arthroplasty and/or in combination with a fracture were excluded.

Risk factors regarding QTR were not incorporated in the selection process. This was in accordance with risk factors not affecting the postoperative outcome $(21,24)$.

Both uni- and bilateral ruptures were included. As reported by Popov et al (22), this was another factor that could possibly affect the results. Bilateral ruptures were found to have better outcomes than unilateral. Due to the limited number of studies, this study examined both bi- and unilateral QTR. Studies including less than 8 patients were excluded based on being a multi case study, and therefore not relevant here due to the descriptive nature of the data. Studies with an insufficient description of their method and/or their results were also excluded due to an unclear outcome of the specific surgical approach. This study demonstrates that only a few studies with proper design and sufficient statistical power have been carried out on the defined surgical procedures, and even fewer gives useful and well-defined outcomes.

The two patient groups allocated according to their surgical repair method, transosseous sutures or anchors fixation, were comparable regarding gender, age and time to followup. QTR patients included were mostly men above the age of 50. This was consistent with previous findings (4).

Only postoperative ROM averages were reported. In total $123^{\circ}$ in 81 QTRs in the anchor group and $138^{\circ}$ in 8 QTRs in the transosseous group with a further 2 out of 15 QTRs being $<120^{\circ}$. As $120^{\circ}$ is considered a sufficient ROM and a good postoperative result $(31,34)$, the groups seem comparable. Similar results were seen in the studies of Boudissa et al. (26), with ROM postoperatively being $133^{\circ}$ in 25 QTRs, and Wenzl et al. (35) with a ROM of $131,7^{\circ}$ in 29 patients. However with both these studies using 2-3 different surgical techniques, and with transosseous sutures and end-to-end sutures used in both studies, the results are not completely comparable to the studies included here.
3 extension lags were reported in both groups, respectively, 3 out of 45 QTRs $(6,7 \%)$ in the anchor fixation group, and 3 out of 23 QTRs (13\%) in the transosseous sutures group. This difference has earlier been proposed as being due to a difference in postoperative rehabilitation protocol(35). The two groups differed in this with the anchor fixation group implementing early passive motion and partial weight bearing for the first 6 weeks after surgery (29). Furthermore, increasing restricted active motion was allowed from week 3. The transosseous group immobilized their patients in a cast or knee brace for 4,5-6 weeks. Both Ramseier et al.(25) and West et al.(36) looked at early rehabilitation vs immobilization. No significant postoperative difference in ROM and re-rupture was seen.

2 re-ruptures in 45 QTRs $(2,5 \%)$ were reported in the anchor group compared to 0 in 23 QTRs $(0 \%)$ in the transosseous group. This did to a certain extent correlate with previous findings(1), stating a $2 \%$ postoperative re-rupture risk. The included re-ruptures in the anchor fixation group, were excluded by Mille et al (28) due to that the two cases were considered technical failures. These two patients did not wear the splint as instructed. Other postoperative complications and the need for reoperation were not taken in to account in this study.

Lysholm score was reported in both groups with an average of 88,9 in the anchor fixation group and 94 in the transosseous group, respectively. As reported by Briggs et al. (37), the average score in the general population is 94 out of 100 . However, with the limited number of studies, being further limited in the transosseous group with only Plesser et al. (29) reporting the score, there is not enough available data to conclude on the Lysholm Score.

The Lysholm score was chosen as outcome measure in spite of being an overall knee score (19) and not being specific for QT. However, given the fact that no QTR specific score has been developed, it is a useful and easy-to-use starting point.

The main weakness this study is a lack of studies with a high level of evidence. No randomized controlled trials were identified. This presented us with results which were difficult to interpret. There was not sufficient data for carrying out a meta-analysis, or even for simple statistical comparisons. The Coleman Methodology Score shows a general possibility of bias and confounding in the included studies with only one study scoring $>55$ out of 100 . Furthermore, in all of the included studies patient compliance to the postoperative protocol was not stated or discussed, resulting in a score of 0 in the category. Likewise, whether the investigator was independent of the surgeon or not, and whether the assessment by the subjects themselves was with minimal investigator assistance was not stated or discussed in most 
of the included articles. These were therefore given a score of 0 in both minor CMS categories.

Since most of the studies were retrospective, the diagnostic certainty was assessed as adequate, due to only subjects suffering from QT being included and the diagnosis being confirmed during surgery.

Based on present research and evidence we were not able to asses if there is a postoperative advantage when using suture anchors instead of transosseous sutures for QTR. Future investigations into the subject must be better protocolized to give higher evidence by comparing the two methods directly, best in a randomized controlled study. Then clear guidelines can be given for the surgical procedures for QTR.

\section{REFERENCES}

1. CirielloV, GudipatiS, Tosounidis T, Soucacos PN, GiannoudisP V. Clinical outcomes after repair of quadriceps tendon rupture: A systematic review. Injury [Internet]. 2012;43(11):1931-8. Available from: http://dx.doi.org/10.1016/j.injury.2012.08.044

2. Shah M, Jooma N. Simultaneous bilateral quadriceps tendon rupture while playing basketball. $\mathrm{Br} \mathrm{J}$ Sports Med. 2002;36(2):152-3.

3. Kannus P, Natri A. Etiology and pathophysiology of tendon ruptures in sports. Scand J Med Sci Sports. 1997;7(2):107-12.

4. Shah MK. Simultaneous bilateral rupture of quadriceps tendons: analysis of risk factors and associations. South Med J [Internet]. 2002;95(8):860-6. Available from: http://www. ncbi.nlm.nih.gov/pubmed/12190222

5. Clayton RAE, Court-Brown CM. The epidemiology of musculoskeletal tendinous and ligamentous injuries. Injury. 2008;39(12):1338-44.

6. Lyle J, LA C. Quadriceps tendon rupture. eMedicine Spec Orthop Surgery, Knee. 2005;1-11.

7. Scuderi C. Ruptures of the quadriceps tendon: Study of twenty tendon ruptures. Am J Surg. 1958;95(I):626-35.

8. Neubauer T, Wagner M, Potschka T, Riedl M. Bilateral, simultaneous rupture of the quadriceps tendon: A diagnostic pitfall? Rort of three cases and meta-analysis of the literature. Knee Surgery, Sport Traumatol Arthrosc. 2007;15(1):43-53.

9. Levy M, Goldstein J, Rosner M. A Method of Repair for Quadriceps Tendon or Patellar Ligament (Tendon) Ruptures Without Cast Immobilization. Clin Orthop Relat Res. 1987;218:297-301.

10. Maffulli N, Papalia R, Torre G, Denaro V. Surgical Treatment for Failure of Repair of Patellar and Quadriceps Tendon Rupture With Ipsilateral Hamstring. Sport Med Arthrosc Rev. 2017;25(1):51-5.

11. Leopardi P, Vico G Di, Rosa D, Cigala F, Maffulli N. Reconstruction of a chronic quadriceps tendon tear in a body builder. Knee Surgery, Sport Traumatol Arthrosc. 2006;14(10):1007-11.

12. McLaughlin HL, Francis C. Operative Repair of Injuries to the Quadriceps Extensor Mechanism. 1956;

13. Brossard P, Le Roux G, Vasse B. Acute quadriceps tendon rupture repaired by suture anchors: Outcomes at 7 years' follow-up in 25 cases. Orthop Traumatol Surg Res. 2017 Jun;103(4):597-601.

\section{CONCLUSION}

The present study implies a slight postoperative advantage when using transosseous sutures compared to anchor fixation based on a better percentage in extension lag. The transosseous fixation group was superior in postoperative ROM, re-ruptures and Lysholm score. However, the evidence is low due to the limited number of available studies and the quality of these.

\section{Conflicts of interest}

The authors have no affiliations with or involvement in any organization or entity with any financial interest in the topic (38).

14. Maniscalco P, Bertone C, Rivera F, Bocchi L. A new method of repair for quadriceps tendon ruptures. A case report [Internet]. Vol. 42, Panminerva medica. 2000. p. 223-5. Available from: http://ovidsp.ovid.com/ovidweb.cgi? T=JS\&PAGE=reference $\& \mathrm{D}=$ emed $5 \& \mathrm{NEWS}=\mathrm{N} \& \mathrm{AN}=11218630$

15. Lighthart, William A, MD; Cohen, David A, MD; Levine, Richard G, MD; Parks, Brent G, MSc; Boucher, Henry R M. Suture Anchor Versus Suture Through Tunnel Fixation for Quadriceps Tendon Rupture: A Biomechanical Study. Organ Dev J. 2012;(February).

16. Petri M, Dratzidis A, Brand S, Calliess T, Hurschler C, Krettek C, et al. Suture anchor repair yields better biomechanical properties than transosseous sutures in ruptured quadriceps tendons. Knee Surg Sports Traumatol Arthrosc. 2015;23(4):1039-45.

17. Bushnell BD, Whitener GB, Rubright JH, Creighton RA, Logel KJ, Wood ML. The use of suture anchors to repair the ruptured quadriceps tendon. J Orthop Trauma. 2007;21(6):407-13.

18. Harris JD, Abrams GD, Yanke AB, Hellman MD, Erickson BJ, Bach Jr BR. Suture anchor repair of quadriceps tendon rupture. Orthopedics [Internet]. 2014;37(3):183-6. Available from: http://www.healio.com/ /media/Journals/ ORTHO/2014/3_March/10_3928_01477447_20140225_0 6/ORTHO0314HARRIS.pdf\%5Cnhttp://ovidsp.ovid.com/ ovidweb.cgi? $\mathrm{T}=\mathrm{JS} \& \mathrm{PAGE}=$ reference $\& \mathrm{D}=$ emed $16 \& \mathrm{NEWS}=$ $\mathrm{N} \& A N=372674137$

19. Lysholm J, Gillquist J. Evaluation of knee ligament surgery results with special emphasis on use of a scoring scale. Am J Sports Med [Internet]. 1982;10(3):150-4. Available from: http://www.ncbi.nlm.nih.gov/entrez/query.fcgi? cmd=Retrieve $\& \mathrm{db}=$ PubMed \&dopt $=$ Citation\&list_uids $=6896798$

20. Coleman BD, Khan KM, Maffulli N, Cook JL, Wark JD. Studies of surgical outcome after patellar tendinopathy: clinical significance of methodological deficiencies and guidelines for future studies. Victorian Institute of Sport Tendon Study Group. Scand J Med Sci Sport. 2000;10(1):2-11.

21. Konrath GA, Chen D, Lock T, Goitz HT, Watson JT, Moed $\mathrm{BR}$, et al. Outcomes following repair of quadriceps tendon ruptures. J Orthop Trauma. 1998;12:273-9.

22. Popov I, Ristic V, Maljanovic M, Milankov V. Quadriceps tendon rupture - treatment results. Med Pregl. 2013;66(11-12):453-8. 
23. Vainionpää S, Böstman O, Pätiälä H, Rokkanen P. Rupture of the quadriceps tendon. Acta Orthop Scand. 1985;56:433-5.

24. O'Shea K, Kenny P, Donovan J, Condon F, McElwain JP. Outcomes following quadriceps tendon ruptures. Injury. 2002;33(3):257-60.

25. Ramseier LE, Werner CML, Heinzelmann M. Quadriceps and patellar tendon rupture. Injury. 2006;37(6):516-9.

26. Boudissa M, Roudet A, Rubens-Duval B, Chaussard C, Saragaglia D. Acute quadriceps tendon ruptures: A series of 50knees with an average follow-up of more than 6 years. Orthop Traumatol Surg Res [Internet]. 2014;100(2):213-6. Available from: http://dx.doi.org/10.1016/j.otsr.2013.09.014

27. Rasul AT, Fischer DA. Primary repair of quadriceps tendon ruptures. Results of treatment. Clinical Orthopaedics and Related Research; 1993. p. 205-7.

28. Mille F, Adam A, Aubry S, Leclerc G, Ghislandi X. Prospective multicentre study of the clinical and functional outcomes following quadriceps tendon repair with suture anchors. Eur J Orthop Surg Traumatol. 2015;26(1):85-92.

29. Plesser S, Keilani M, Vekszler G, Hasenoehrl T, Palma S, Reschl M, et al. Clinical outcomes after treatment of quadriceps tendon ruptures show equal results independent of suture anchor or transosseus repair technique used - A pilot study. PLoS One. 2018;13(3):e0194376.

30. Huleatt J, Gebrelul A, Premkumar A, Xerogeanes J. Suture Anchor Repair of Quadriceps Tendon and Patellar Tendon Ruptures. Tech Orthop. 2018;00(00).

31. De Baere T, Geulette B, Manche E, Barras L. Functional results after surgical repair of quadriceps tendon rupture. Acta Orthop Belg. 2002;68(2):146-9.
32. Brossard P, Le Roux G, Vasse B. Acute quadriceps tendon rupture repaired by suture anchors: Outcomes at 7 years' follow-up in 25 cases. Orthop Traumatol Surg Res [Internet]. 2017; Available from: http://linkinghub.elsevier.com/retrieve/ pii/S1877056817300981

33. Chaudhry S, Dehne K, Hussain F. A review of suture anchors. Orthop Trauma [Internet]. 2017;1-8. Available from: http:// dx.doi.org/10.1016/j.mporth.2016.12.001

34. Siwek CW, Rao JP. Ruptures of the Extensor Mechanism of the Knee Joint. The Journal of Bone and Joint Surgery; 1981. p. 932-7.

35. Wenzl ME, Kirchner R, Seide K, Strametz S, Jürgens C. Quadriceps tendon ruptures-is there a complete functional restitution? Injury [Internet]. 2004;35(9):922-6. Available from: http://www.sciencedirect.com/science/article/pii/ S0020138303002614

36. West JL, Keene JS, Kaplan LD. Early motion after quadriceps and patellar tendon repairs: outcomes with single-suture augmentation. Am J Sports Med [Internet]. 2008;36(2):31623. Available from: http://ajs.sagepub.com/lookup/ doi/10.1177/0363546507308192\%5Cnhttp://www.ncbi.nlm. nih.gov/pubmed/17932403

37. Briggs KK, Steadman JR, Hay CJ, Hines SL. Lysholm score and tegner activity level in individuals with normal knees. Am J Sports Med. 2009;37(5):898-901.

38. Padulo J, Oliva F, Frizziero A, Maffulli N, Padulo J. Muscles, Ligaments and Tendons Journal - Basic principles and recommendations in clinical and field Science Research: 2016 Update Corresponding author : 2016;1-5. 